\title{
THE CUSTOMER IS NOT ALWAYS RIGHT, BUT ARE YOUR EMPLOYEES?
}

\author{
Gauthier Catherine, HEC Montréal, Canada \\ Chebat Jean-Charles, HEC Montréal, Canada \\ Vandenberghe Christian, HEC Montréal, Canada
}

\begin{abstract}
The customer might not always be right, but he has more power then he has ever had in the past. If two decades ago, "word of mouth" meant calling friends to discuss a bad experience, Internet has given the power to the customer to express their dissatisfaction to a larger audience.
\end{abstract}

There are plenty of reasons why customers can be dissatisfied (i.e product defects, delays, employee behavior, etc.). The important fact about customer dissatisfaction is that the customers do not always express their discontent to the company. From those who decide to complain, Naylor (2003) estimated that fewer than $50 \%$ of complaints received a response from the company. And from those who could be answered, Lewis and McCann (2004) in a study of Hotel complaint handling, showed that only $50 \%$ of customers were satisfied or very satisfied with how the company dealt with the problem. From this last statistic, we can recall that complaint handling has been proven to be a major source of "switching behavior" (Smith \& Bolton, 2002).

In his interaction with the company, the customer is facing a customer service representative (CSR) who is ultimately responsible for the service delivery and recovery. Those employees are, in a customer's perspective, a materialization of the organization (Zeithaml \& Bitner, 2000). Coming with different personalities, from different cultural backgrounds and work experiences, those employees are sandwiched between management and the customer with a goal of behaving to provide customer satisfaction.

It is important to note that there are many people working in call centers worldwide: over four million in United States, eighthundred thousand in the United Kingdom, around half a million in Canada and also in the subcontinent, and the list goes on in many other countries (in Russell, 2009 p.5-6). Our study will be held in Canada, and at least 200 agents and their related supervisors will be questioned.

The purpose of this study is to analyze the behavior, more specifically pro-social and service sabotage behaviors of customer service representatives, in a call center context, where the employee must resolve critical incidents that happen between the company and the customer. We examine how managerial practices of empowerment (relational empowerment) are internalized by employee (psychological empowerment), and provoke those positive or negative behaviors. We include in our model, commitment toward organization as well as a cultural factor of Triandis (1998), as moderating factors.

Ultimately, the goal of this study was, in the first place, to shed light on the role of managerial practices in call centers in shaping the employee's behavior toward customers. Additionally, this study also aimed to explore the mechanisms of cultural differences that affect frontline employees' perception of those managerial practices and of the achievement of positive and negative behavior. Knowing immigration is a worldwide phenomenon; managers must understand how employees from different cultures incorporate those practices. We wish to prove that empowerment of customer service representatives will affect pro- social behavior, which will ultimately affect customer satisfaction and first call resolution, which is a well known $K P I$ for call centers. When looking at negative behavior, we estimate that empowerment and commitment would reduce service sabotage behavior. The outcome of this research will be to help academic as well as researchers understand better the connection between those four concepts (empowerment, commitment, culture and behavior) as well as the importance of the call center as an organizational competitive advantage.

References available upon request 